\title{
Automatic EOG Artifact Removal in Brain-computer Interface Systems
}

Wei-Yen Hsu*, Cheng-Xuan Li, Meng-Chen Li, Hui-Yu Tien

Department of Information Management

National Chung Cheng University

Chiayi, Taiwan

shenswy@gmail.com

shenswy@mis.ccu.edu.tw

ABSTRACT: In this study, we propose a system to recognize the finger-lifting electroencephalogram (EEG) data. Combined with independent component analysis (ICA) and feature extraction, fuzzy c-means (FCM) clustering is used to discriminate between left and right finger movement without supervision. ICA is used to eliminate the electrooculography (EOG) artifacts. Wavelet-fractal features are then extracted from wavelet data via fractal dimension. FCM clustering is used for feature discrimination. It is an unsupervised approach suitable for the applications of biomedical signals. After EOG artifact removal, the performance is improved for all subjects.

Keywords: Electroencephalogram (EEG), Independent Component Analysis (ICA), Wavelet Transform (WT), Fuzzy C-means (FCM), Brain-Computer Interface (BCI)

Received: 9 May 2018, Revised 17 June 2018, Accepted 29 June 2018

DOI: $10.6025 / \mathrm{jmpt} / 2018 / 9 / 4 / 117-123$

(C) 2018 DLINE. All Rights Reserved

\section{Introduction}

Brain-computer interface (BCI) is a communication system that provides an alternative channel from human brain to computers by analyzing the brain's mental activities [1-6]. Electroencephalographic (EEG) analysis focuses on discriminating left finger movement from right one.

Independent component analysis (ICA) is a statistical method for transforming observed multidimensional mixed signals into components that are statistically as independent as possible. It is a blind source separation method, which estimates the source components under unknown knowledge of sources. In contrast to principal component analysis (PCA), only ensuring output patterns are uncorrelated, ICA makes certain their statistical independence. In this study, the FastICA algorithm [7] is adopted to eliminate the electrooculography (EOG) artifacts.

Fractal dimension is common fractal features, which has been applied to various biomedical signal analyses, such as seizure

Journal of Multimedia Processing and Technologies Volume 9 Number 4 December 2018 
onset detection in epilepsy [8] and image coding [9]. We use DWT to decompose EEG signals into multiscales, and then obtain multiresolution fractal feature vectors (MFFVs) from multiscale EEG signals. As MFFVs have been shown to achieve promising results in finger movement applications [10], we have opted to use MFFVs as features in this study. Fuzzy c-means clustering is an unsupervised approach that partitions a collection of feature vectors into a number of subgroups based on minimizing the trace of a within-cluster scatter matrix [11-13]. Therefore, we use FCM clustering for the classification of EEG data in this study.

This paper is organized as follows: In Section 2, EEG data acquisition and analysis are presented; Section 3 describes experimental results and discussions on single-trial ERP data; finally, conclusions are given in Section 4.

\section{Methods}

\subsection{Data Acquisition}

EEG signals were recorded from four untrained subjects (three males and one female, two left-handed and two right-handed) in a shielded room using 13 silver/silver chloride electrodes. As illustrated in Figure 1.

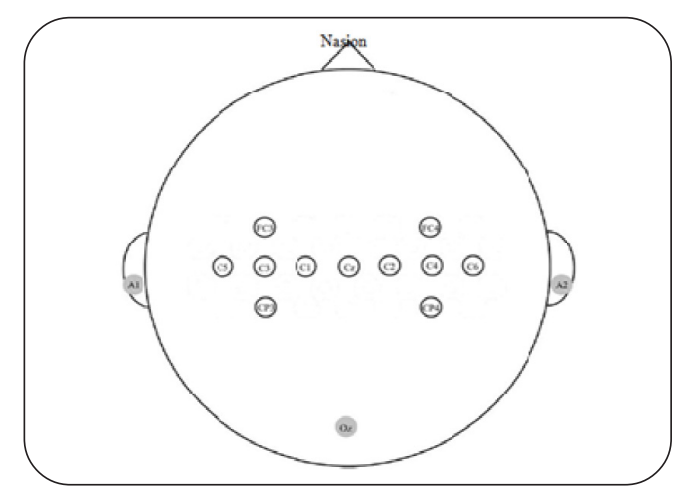

Figure 1. International EEG electrode 10-20 systems

They included ten scalp EEG channels (C3, C5, FC3, C1, CP3, C4, C2, FC4, C6, and CP4), two EMG channels for monitoring left and right muscle activity, and one channel on the forehead to record possible EOG artifacts and eye blinks during the experiment [14]. All electrodes were referenced to the A1 lead at the left earlobe. During the experiments, each subject was asked to perform two trials, which included left and right finger lifting in each test. Each trial was ten seconds in length; therefore, it took twenty seconds in a test. For each lifting trial, the first $4 \mathrm{~s}$ was quiet and then an acoustic stimulus was given as a cue to signify the beginning of left or right finger lifting. At the same time, each subject was asked to execute a finger lifting. We recorded sixty tests for each subject. Data segments of the finger lifting were acquired from second -2 to second 2 , where second 0 stands for the trigger of movement by detecting the peak EMG signal after linear envelope processing.; footnotes should be avoided. An informative abstract of 200 words or less should be included on the first page.

\subsection{EOGArtifact Removal}

ICA is a statistical method for transforming observed multidimensional mixed signals into components that are statistically as independent from others as possible. Statistical independence needs that all high-order correlations are zero, while decorrelation only minimnd-order statistics. The application of ICA for blind source separation of EEG signals is based on a reasonable assumption that EEG data acquired from multiple scalp electrodes are the linear combinations of temporally independent components.

In this study, the FastICA algorithm [7] is adopted to eliminate the EOG artifacts due to the characteristic of its fast convergence. It removes the means of row vectors from the matrix and then uses a whitening procedure to transform the covariance matrix of zero-mean data into an identity matrix. Finally, it separates the whitened data into a set of components which are as mutually independent as possible. After the removal of EOG artifacts, the EEG signals without EOG artifacts are recovered from remaining independent components. The procedure for the elimination of EOG artifacts is shown in Figure 2. In Figure 2(a), acquired (mixed) EEG signals are given. Figure 2(b) shows independent components after performing the FastICA algorithm. The recovered EEG signals without EOG artifacts are shown in Figure 2(c).

118 Journal of Multimedia Processing and Technologies Volume 9 Number 4 December 2018




\subsection{Data Preprocessing}

The Laplacian filter is a simple but effective filtering method [15]. It calculates the second derivative of the spatial voltage distribution for a selected electrode. It is a high-pass spatial filter that enhances localized activities and reduces background noise. This filter is achieved by subtracting the average potential of a set of surrounding electrodes from the electrode of interest:

$$
V_{i}^{\text {Lap }}=V_{i}-\frac{1}{N} \sum_{j \in S_{i}} V_{j}
$$

where $V_{i}$ represents the potential between the $i^{\text {th }}$ electrode and the reference A1, and $S_{i}$ and $N$ stand for the set of electrodes surrounding the $i^{\text {th }}$ electrode and the number of surrounding electrodes, respectively.

\subsection{Feature Extraction}

Feature extraction is performed rather than directly classifying tizes the secohe native EEG data. We performed the discrete wavelet transform and fractal dimension on the filtered active segments. A signal is characterized with the formulation by decomposing it into sub-bands, and each sub-band can be treated individually based on its characteristics. Multiresolution representation of the filtered active segments is achieved by discrete wavelet transform (DWT). Fractal dimension (FD) is one of the most popular fractal features, and we opted to use the FD due to the fact that it is relatively insensitive to signal scaling and shows a strong correlation with human judgment of surface roughness [16]. The multiresolution fractal feature vectors (MFFVs) are formed by concatenating various-scale fractal features, calculated from the active segment itself and all of its subbands using the modified fractal dimension.

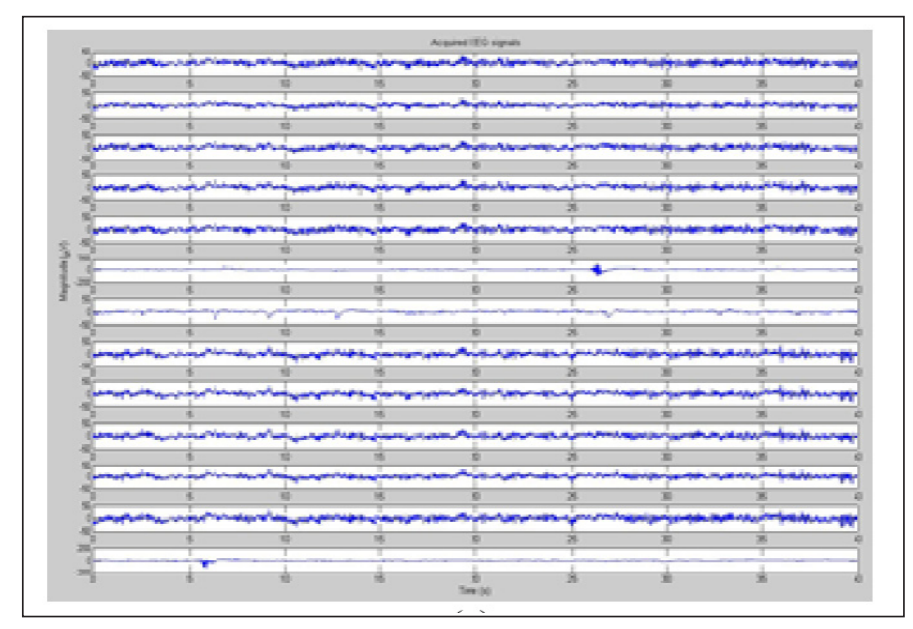

(a)

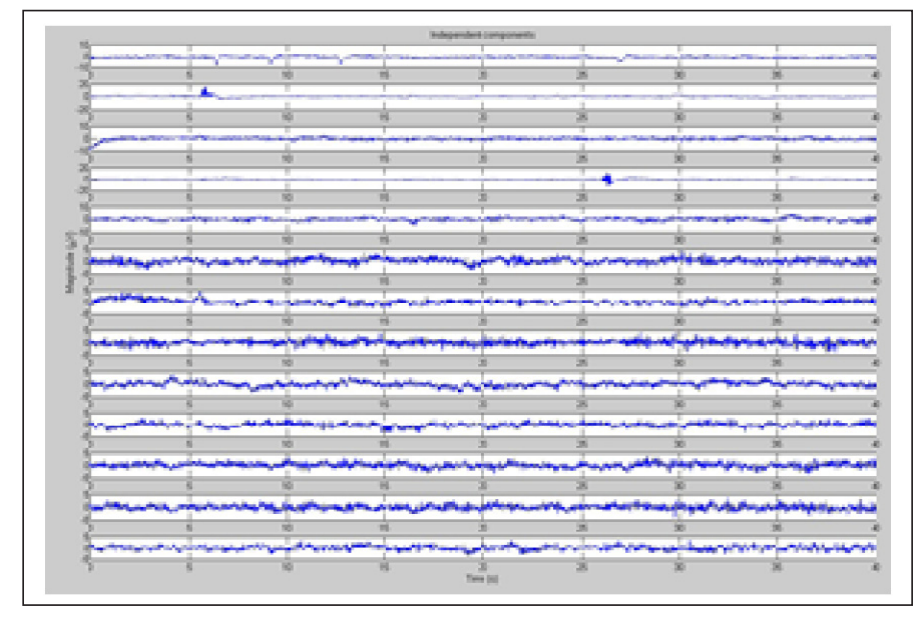

(b) 


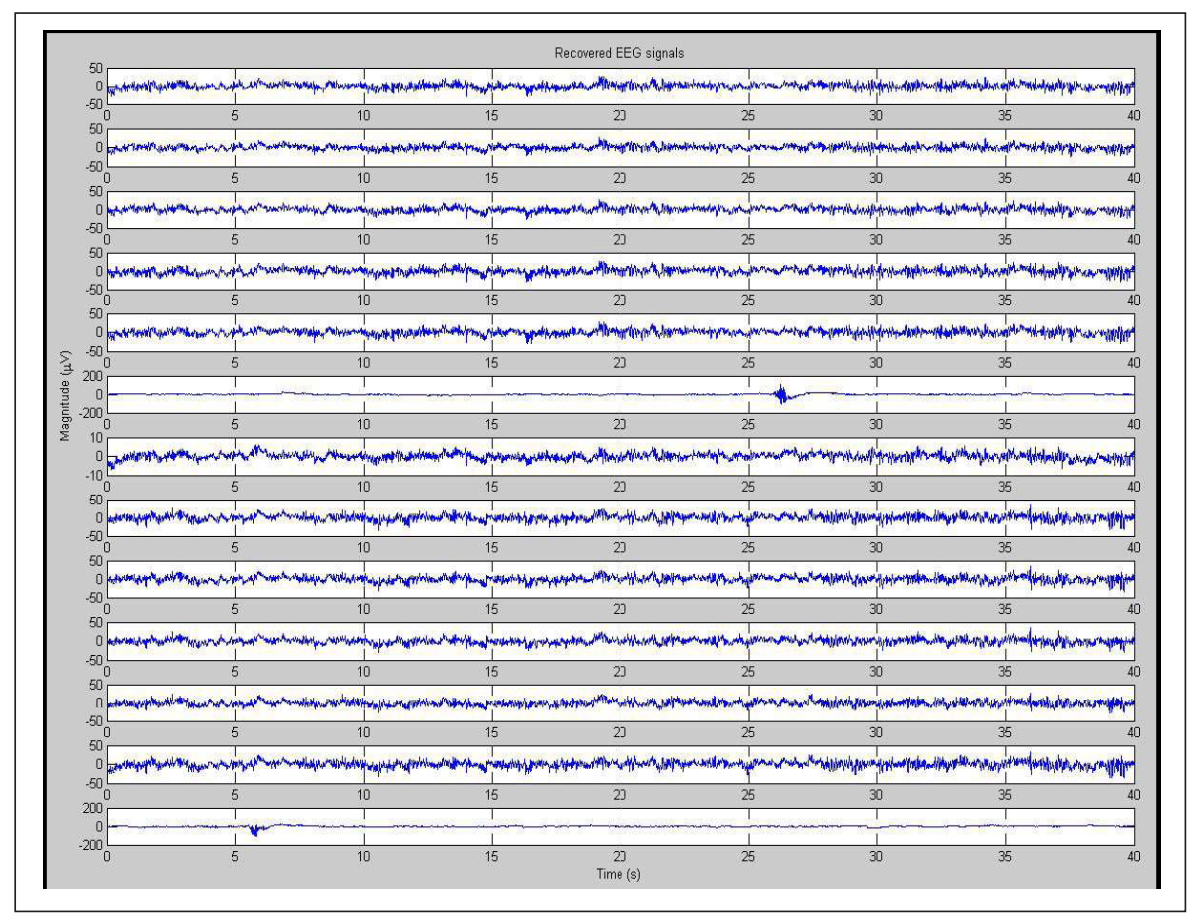

(c)

Figure 2. Procedure for EOG artifact elimination. (a) Acquired EEG signals. (b) Independent components.

(c) Recovered EEG signals without EOG artifacts

\subsection{Fuzzy c-means Clustering}

Clustering is a process for classifying training samples in such a way that samples within a cluster are more similar to one another than samples belonging to different clusters. Similarity measures employed to classify samples depend on the object characteristics e.g. distance, vector, entropy, etc. Many clustering approaches have also been demonstrated such as the hard clustering algorithm $[17,18]$ and the soft (fuzzy) clustering algorithm, each of which has its own special characteristics. There are many applications in clustering strategy $[19,20]$. The fuzzy clustering method assigns the sample with a number, $\mathrm{m}$, between zero and one described as a membership function. In this paper, fuzzy clustering method is to classify the feature vectors extracted from the original EEG data, and recognize complicated brain mental tasks, such as left and right finger movement.

The FCM clustering algorithm was first introduced by Dunn [21], the related formulations and algorithms were extended by Bezdek [22]. The FCM approach, like the conventional clustering techniques, minimizes an objective function in the least squared error sense. For class number $c(c \geq 2)$, sample number $n$ and fuzzification parameter $m(1 \leq m<\infty)$, the algorithm chooses $u_{i}: X$ $\rightarrow[0,1]$ so that $\sum_{i} u_{i}=1$ and $w_{j} \in R^{d}$ for $j=1,2, \ldots, c$ to minimize the objective function.

$$
J_{F C M}=\frac{1}{2} \sum_{j=1}^{c} \sum_{i=1}^{n}\left(u_{i, j}\right)^{n}\left\|x_{i}-w_{j}\right\|^{2}
$$

Where $u_{i, j}$, is the value of $j^{\text {th }}$ membership grade on $i^{\text {th }}$ sample $x_{i}$. The cluster centroids $w_{1}, \ldots, w_{j}, \ldots, w_{c}$ can be regarded as prototypes for the clusters represented by the membership grades. For the purpose of minimizing the objective function, the cluster centroids and membership grades are chosen so that a high degree of membership occurs for samples close to the corresponding cluster centroids. The steps of the FCM algorithm are listed below.

Step 1: Initialize the cluster centroids $w_{j}(2<j<c)$, fuzzification parameter $m(1<m<\infty)$, and the value $\varepsilon>0$. Give a fuzzy cpartition $U^{(0)}$ and $t=1$.

$120 \quad$ Journal of Multimedia Processing and Technologies Volume 9 Number 4 December 2018


Step 2: Calculate the membership matrix $U^{(t)}=\left[u_{i, j}\right]$ by

$$
u_{i, j}=\frac{\left(\frac{1}{\left(d_{i, j}\right)^{2}}\right)^{1 /(m-1)}}{\sum_{l=1}^{c}\left(\frac{1}{\left(d_{l, i}\right)^{2}}\right)^{1 /(m-1)}}
$$

where $d_{i, j}$, is the Euclidean distance between the training sample $x_{i}$ and the class centroid $w_{j}$.

Step 3: Update the class centroids by

$$
w_{j}=\frac{1}{\sum_{i=1}^{n}\left(u_{i, j}\right)^{m}} \sum_{i=1}^{n}\left(u_{i, j}\right)^{m} x_{i} \text { for every } j
$$

Step 4: Compute $\Delta=\max \left(\left|U^{(t+1)}-U^{(t)}\right|\right)$. If $\Delta>\varepsilon, t=t+1$ and go to step 2 ; otherwise stop the process.

\section{Results and Discussion}

An unsupervised method utilizing FCM clustering is proposed for left and right finger movement classification from single-trial EEG data in this study. The FCM algorithm is implemented to recognize left and right finger movement data without any training in advance. As FCM clustering is an unsupervised approach that partitions a collection of feature vectors into a number of subgroups based on minimizing the trace of a within-cluster scatter matrix, it is adopted to automatically classify MFFVs into two clusters, left and right finger movement.

An experiment for evaluating the performance of EOG artifact removal was performed. Table shows the comparisons of classification accuracy without and with EOG artifact removal. We observe that classification with EOG artifact removal gives the better results for all subjects.

\begin{tabular}{|c|l|l|l|l|l|}
\hline Classification Accuracy [\%] & Subject 1 & Subject 2 & Subject 3 & Subject 4(FCM) & Average \\
\hline without EOG artifact removal & 72.4 & 75.3 & 61.5 & 61.0 & 67.6 \\
\hline with EOG artifact removal & 76.1 & 81.9 & 63.2 & 64.8 & 71.5 \\
\hline
\end{tabular}

Table 1. Comparison of classification accuracy between without and with EOG artifact removal

\section{Conclusion}

In this study, we propose an EEG analysis system for the single-trial recognition of EEG data. Fuzzy c-means clustering together with ICA and feature extraction are applied for the recognition of left and right finger movement. ICA is used to eliminate the EOG artifacts. The wavelet-fractal features are obtained using fractal dimension extracted from the DWT data. They both can improve the classification of mental tasks. FCM clustering is used for the classification of the EEG data. FCM clustering is a robust approach suitable for the classification of non-stationary EEG signals. The experimental results demonstrate that ICA is a promising approach to eliminate the EOG artifacts.

\section{Acknowledgments}

The authors would like to express their sincerely appreciation for grants from MOST105-2410-H-194-059-MY3, Ministry of 
Science and Technology, Taiwan.

\section{References}

[1] Hyvarinen, A., Karhunen, J., Oja, E. (2001). Independent Component Analysis. New York: John Wieley \& Sons, Inc.

[2] Accardo, A. P., Affinito, M., Carrozzi, M., Cisint, S., Bouquet. F. (1998). Comparison between spectral and fractal EEG analyses of sleeping newborns, In: Proceedings 20th Annual International Conference of the IEEE Engineering in Medicine and Biology Society, $3,1569-1571$.

[3] Pentland, A. P. (1984). Fractal based description of natural scenes, IEEE Transactions on Pattern Analysis and Machine Intelligence. 6, 661-674.

[4] Brunner, C., Naeem, M., Leeb, R., Graimann, B., Pfurtscheller, G. (2007). Spatial filtering and selection of optimized components in four class motor imagery EEG data using independent components analysis, Pattern Recognition Letters, 28 (8) 957-964.

[5] Lin, C. Y., Chen, C. H. (2007). An Invisible Hybrid Color Image System Using Spread Vector Quantization Neural Networks with Penalized FCM. Pattern Recognition, 40 (6) 1685-1694.

[6] Balakrishnan, D., Puthusserypady, S. (2005). Multilayer perceptrons for the classification of brain computer interface data. In: Proceedings IEEE 31st Annual Northeast Bioengineering Conference.

[7] Pfurtscheller, G., Neuper, C., Guger, C., Harkam, W., Ramoser, R., Schlögl, A., Obermaier, B., Pregenzer, M. (2000). Current trends in Graz brain-computer interface (BCI) research, IEEE Transactions on Rehabilitation Engineering, 8 (2) 216-219.

[8] Bezdek, J. C. (1973). Fuzzy mathematics in Pattern Classification, Ph.D. Dissertation, Applied Mathematics, New York: Ithaca, Cornell University.

[9] Dunn, J. C. (1974). A fuzzy Relative of the ISODATA process and its use in detecting compact wellseparated clusters. Journal of Cybernetics, 3 (3) 32-57.

[10] Jasper H. (1958). The ten-twenty electrode system of the international federation. Electroenceph. Clin. Neurophysiol 10. $371-5.140$

[11] Millán, Jd.R., Renkens, F., Mouriñ, J., Gerstner, W. (2004). Noninvasive brain-actuated control of a mobile robot by human EEG, IEEE Transactions on Biomedical Engineering, 51(6) 1026-1033.

[12] Shinkai, K. (2008). Decision analysis of fuzzy partition tree applying fuzzy theory. International Journal of Innovative Computing, Information and Control, 4(10) 2581-2594.

[13] Bobrowski, L., Bezdek, J. C. (1991). C-means clustering with the $\imath_{1}$ and $\imath_{\propto}$ norms. IEEE Transactions on Systems, Man, and Cybernetics, 21, 545-554.

[14] Parra, L., Alvino, C., Tang, A. C., Pearlmutter, B. A., Yeung, N., Osman, A., Sajda, P. (2002). Linear spatial integration for single trial detection in encephalography, NeuroImage 7 (1) 223-230.

[15] Ismail, M. A., Selim, S. Z. (1986). Fuzzy c-mean: Optimality of solutions and effective termination of the algorithm, Pattern Recognition, 19 (6) 481-485.

[16] Kirlangic, M. E., Perez, D., Kudryavtseva, S., Griessbach, G., Henning, G., Ivanova, G. (2001). Fractal dimension as a feature for adaptive electroencephalogram segmentation in epilepsy, In: Proceedings of the 23rd Annual International Conference of the IEEE Engineering in Medicine and Biology Society, 2, 1573-1576.

[17] McFarland D. J., McCane, L. M., David, S. V., Wolpaw, J. R. (1997). Spatial filter selection for EEG-based communication. Electroencephalogr. Clin. Neurophysiol 103:386-94.

[18] Yin, P. Y., Chen, L. H. (1994). A new non-iterative approach for clustering. Pattern Recognition Letters, 15, p.125-133.

[19] Kuo, W. F., Lin, C. Y., Sun, Y. N. (2008). Brain MR Images Segmentation Using Statistical Ratio: Mapping between Watershed and Competitive Hopfield Clustering Network Algorithms. Computer Methods and Programs in Biomedicine, 91(3) 191-198. (September).

[20] Hsu, W. Y., Sun, Y. N. (2009). EEG-based motor imagery analysis using weighted wavelet transform features, Journal of Neuroscience Methods, 176(2,.310-318. 
[21] Hsu, W. Y., Poon, P. W. F., Sun, Y. N. (2008). Automatic seamless mosaicing of microscopic images: enhancing appearance with colour degradation compensation and wavelet-based blending, Journal of Microscopy, 231(3) 408-418.

[22] Hsu, W. Y., Lin, C. C., M. S. Ju., Sun, Y. N. (2007). Wavelet-based fractal features with active segment selection: Application to single-trial EEG data, Journal of Neuroscience Methods, 163(1) 145-160. 